\title{
A SURVEY ON MOBILITY MODELS FOR VEHICULAR AD HOC NETWORKS
}

\author{
Sanjib Debnath ${ }^{1}$, Abhishek Majumder ${ }^{2}$ \\ ${ }^{1}$ Department of Computer Science and Engineering, Tripura University (A central university) \\ ${ }^{2}$ Department of Computer Science and Engineering, Tripura University (A central university)
}

\begin{abstract}
Vehicular networking is an emerging area of interest in the wireless networking community as well as in the transportation research community. Vehicular ad hoc networks (vanet) are distributed, self-organizing communication networks which progressed from traveling vehicles, and are thus characterized by very high speeds and limited degrees of freedom in node (vehicle) movement patterns. Different mobility models in vehicular ad hoc networks significantly affect the performance of several mobility management protocols. These mobility models imply vehicular movement pattern, street layout, trip selection scheme, path selection scheme, destination selection mechanism, and traffic control mechanism. Therefore a good realistic mobility model is an essential component to evaluate the performance of vanet.
\end{abstract}

Keywords: Ad hoc networks, VANET, Macro mobility, Micro mobility, Mobility model. $* * *$

\section{INTRODUCTION}

VANET is an extreme class of Mobile ad hoc networks (MANET). The main divergence between VANET and MANET is that the nodes (vehicle) do not move in any random direction. Energy is not an issue for VANET because the nodes are already powered. The node contact time is limited, and node speed is bounded by traffic control mechanism. In VANETs, high node mobility and frequent topology changes greatly affect the network performance. Therefore, to maintain seamless connectivity and to provide good performance, mobility management for vehicular networks on different mobility models are very important. The growths of the increased number of vehicles are equipped with wireless transceivers to communicate with other vehicles to form VANETs [1]. VANET involves vehicle-to-vehicle $(\mathrm{V} 2 \mathrm{~V})$ and vehicle-to-infrastructure (V2I) communication.

Vehicle-to-vehicle refers to the direct or multihop communications among vehicles. Due to short range of bandwidth, V2V communication is efficient and cost effective. Vehicle-to-infrastructure refers to the communication between vehicles and infrastructure of Roadside unit (RSU). V2I communications can be used for internet access .Vehicle-tovehicle communication is based on Dedicated Short Range Communication (DSRC). Vehicle-to-infrastructure communication is based on GPRS/3G, WiFi or WiMax. The speed and choice of path defines the highly dynamic topology [2] of VANET. The multihop vehicular ad hoc networks are shown in figure 1. Two different types of data are produced in VANETs are Delay sensitive which is collected by the safety related applications and Delay tolerant which is collected by comfort applications. The main objective of Vehicular networks is to increase safety for drivers and passengers in vehicular scenarios.

\section{OVERVIEW}

Mobility model determines the location of nodes in the topology at any given instant, which in turn directly impacts the network connectivity. To avoid collisions nodes should physically interact with one another. Due to high mobility of vehicles, topologies of VANETs are highly dynamic. The way to visualize mobility model for VANET is to consider it as an ingredient of two blocks: Motion Constraints and Traffic Generator. Motion constraints portray how each vehicle moves and it is obtained from a topological map. Traffic generator, generates different kinds of vehicles and deals with interactions between vehicles. It also generates traffic sign and traffic regulations considerations. Mobility models attempt to mimic the movements [3] of real mobile nodes. Realistic vehicular mobility model is a key component for VANET simulations. A critical aspect in a simulation study of VANETs is the need for a mobility model reflecting the real behavior of vehicular traffic. In VANET, vehicles can only move along roads, motivating the need for a road model. Vehicles do not move independently of each other. They move according to well established vehicular traffic models. 


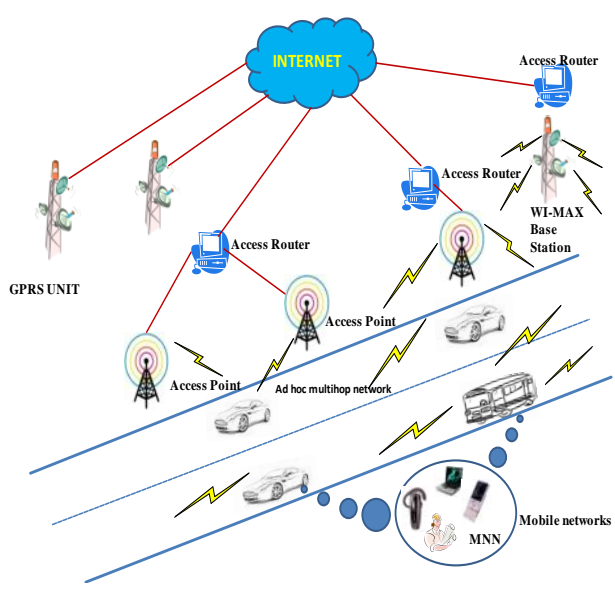

Fig 1: Multihop VANET

\section{FACTORS EFFECTING IN VEHICULAR}

\section{MOBILITY}

There are several factors [4] effecting the mobility of vehicles in vehicular networks. These are:

- Accurate and Realistic topological maps: This kind of maps should manage different densities of roads, contains multiple lanes, different categories of streets and associated velocities.

- Driver route choice: A driver normally has to decide his moving direction at an intersection. Driver can choose to go straight, turn right, or turn left.

- Smooth deceleration and acceleration: Since vehicles do not abruptly break and move, deceleration and acceleration models should be considered.

- Repulsion /Attraction points: Most of the time, drivers are starting from similar initial locations and moving to similar final destinations. Therefore repulsion points and attraction points are not random.

- Vehicles characteristics: Different kinds of vehicles have different characteristics which changes the traffic generation engine when modeling realistic vehicular motions.

- Human driving patterns: Due to human behaviors e.g. mood, sex, age etc. and driving patterns it is very challenging task in realistic simulation.

- Traffic Control Mechanisms: At intersection point the stop signs and traffic lights are the most common traffic control mechanisms.

- Existence of traffic lights: Traffic lights are used to regulate traffic flow moving in different directions. Places where there is a traffic light are likely to have a higher node density. Thus vehicles are forced to stop at the traffic light to wait for the light to turn green.

- Simulation time: Time is another important factor in realistic mobility model. Traffic density is always observed at some peak time of days, such as Rush hours or Special Events.

- Overtaking behavior: A faster vehicle can overtake some other slower ones when overtaking is allowed on a multilane road. Overtaking behavior can have a great effect on the network topology.

\section{VEHICULAR MOBILITY FEATURES}

When dealing with vehicular mobility modeling, two different mobility features [5] are described, i.e., Macro-mobility and Micro-mobility features.

\subsection{Macro-Mobility Features:}

Macro-mobility delineates the road topology, the road structure, the road characteristics, vehicles characteristics and the presence of traffic signs, traffic lights etc. The road structure means unidirectional or bidirectional, single-lane or multi-lane. More important the concept of Macro-mobility admits the effects of the presence of points of interests, which determines movement patterns of different vehicles on the road topology. The different views of macro-mobility [5] are:

\subsubsection{Road Topology Definition}

The length of the streets, the frequency of intersections, the density of buildings can greatly affect important mobility metrics such as the minimum, maximum and average speed of vehicles, or their density over the simulated topological map. VanetMobiSim [6] allows defining the road topology in the following ways:

- User-defined graph: This topology is defined by listing the vertices of the graph and their interconnecting edges.

- GDF map: This topology is imported from a Geographical Data File (GDF) [7].

- Topologically Integrated Geographic Encoding and Referencing (TIGER) map: This topology is extracted from a map obtained from the TIGER [8] database which contains digital descriptions of roads.

- Clustered Voronoi graph: This topology is randomly generated by creating a Voronoi tessellation [9] on a set of non-uniformly distributed points.

\subsubsection{Road Topology Characterization}

Road structure characterization[5], such as multiple lanes or directional traffic flows, speed constraints or intersection crossing rules are important issues to define road topology. VanetMobiSim permits roads with multiple lanes in each direction, physical separation of opposite traffic flows on each road. It also allows definition of independent speed limits on each road topology and traffic signs at each road intersection. Intersections are fully regulated by traffic stop signs, attracting vehicles to stop and wait for free road before crossing. 


\subsubsection{Vehicular Movement Patterns Selection}

VanetMobiSim exploits two different modules [5]: a trip generation module which defines the sets of points of interest, and a path computation module, whose task is to compute the best path between those points. Trip generation module are comprises two different choices i.e. random trip and activity sequences generation. Random trip describes the start and stop points of movement patterns are randomly selected among the vertices of the graph which represents the road topology. Activity sequences describe, in which a set of start and stop points are explicitly provided in the road topology description, and vehicles are forced to move among them. To reach the selected destination the best path selection can be performed in three different ways. The first method uses Dijkstra's algorithm to selects the shortest path to destination. The second method conceives the traffic congestion level, by weighting the cost of traversing an edge also on the number of vehicles traveling on it. The third method covers the first and second method, by reporting for the road speed limit when calculating the cost of an edge, in a way that fastest routes are preferred.

\subsection{Micro-Mobility Features}

Micro-mobility features [5] admit all aspects related to an individual vehicles speed and acceleration modeling. Micro mobility description has an important role to generate realistic vehicular movements. These vehicular movements are responsible for the effects such as smooth speed variation, vehicle queues and traffic jams. Micro mobility model can be classified in three different classes depending on whether the individual speed of vehicles is computed:

- In a deterministic way.

- As a function of nearby vehicles behavior in a single lane scenario.

- As a function of nearby vehicles behavior in a multiflow interaction i.e., urban scenario.

CanuMobiSim [10] provides some mobility models belonging to first class where the speed of each vehicle is determined on the basis of the local state of each vehicle. Those models only work for isolated vehicles. Therefore it fails to reproduce realistic movements of groups of vehicles.

CanuMobiSim also provides Fluid Traffic Model (FTM) [11] and Intelligent Driver Model (IDM) [12] models which belonging to second class. FTM describes vehicle mobility on single lanes, but does not consider the case in which multiple vehicular flows have to interact, as in presence of intersections. The Model also describes the speed as a monotonically decreasing function of the vehicular density. When the traffic congestion reaches a critical state it forces a lower bound on speed, by means of the following equation [5].

$$
s=\max \left[s_{\min }, s_{\max }\left(1-\frac{k}{k_{j a m}}\right)\right]
$$

Where, $\mathrm{s}$ is the output speed, smin and smax are the minimum and maximum speed, kjam is the vehicular density for which a traffic jam is detected, $\mathrm{k}$ is the current vehicular density of the road where the node is, whose speed is being computed. $\mathrm{k}=\mathrm{n} / \mathrm{l}$, where $n$ is the number of vehicles on the road, 1 is the length of the road segment itself.

IDM falls in car following models that characterizes driver's behavior depending on their front vehicle. The instantaneous acceleration of a vehicle is computed according to the following equations [5]:

$$
\begin{gathered}
\frac{d v}{d t}=a\left[1-\left(\frac{v}{v_{0}}\right)^{4}-\left(\frac{s^{*}}{s}\right)^{2}\right] \\
s^{*}=s_{0}+\left(v T+\frac{v \Delta v}{2 \sqrt{a b}}\right)
\end{gathered}
$$

Where, $\mathrm{v}$ is the current speed of the vehicle, $\mathrm{v} 0$ is the desired velocity, $s$ is the distance from preceding vehicle, $\mathrm{s}^{*}$ is the so called desired dynamical distance, s0 is a function of the minimum bumper-to-bumper distance, $\mathrm{T}$ is the minimum safe time headway, $\Delta \mathrm{v}$ is the speed difference with respect to front vehicle velocity, the maximum acceleration and deceleration values are $\mathrm{a}$ and $\mathrm{b}$ respectively.

VanetMobiSim introduced two micro mobility models [5] which belong to the third class. They account for the interaction of multiple converging flows, by acting consistently with the road infrastructure. The first new micro-mobility model is Intelligent Driver Model with Intersection Management (IDMIM). It contributes intersection handling capabilities to the behavior of vehicles driven by the IDM. Two different intersection scenarios in IDM-IM model are: a crossroad regulated by stop signs, or a road junction ruled by traffic lights. In both cases, the model acts only on the first vehicle on each road. Every time a vehicle checks whether there is any intermediate vehicle between it and the point of road intersection. If no vehicle is present, the currently considered vehicle is regulated by the stop sign. Following equations are used by IDM-IM [5].

$$
\begin{gathered}
s=\sigma-S \\
\Delta v=v
\end{gathered}
$$

Where, $\sigma$ is the current distance to the intersection, $S$ is a safety margin, describing the gap between the center of the intersection and the point where the vehicle would actually stop at. In 
macroscopic point of view when a vehicle is stopped at a stop sign, the numbers of vehicles are already waiting to cross the intersection from any of the incoming roads. If there are no other vehicles, the vehicle may pass. Otherwise, the vehicle has to wait for its turn to come under a first-arrived-first-passed and right hand rule policy.

When a vehicle is leading towards a traffic light intersection, it is informed by the macroscopic description. If the traffic light sign is green, the vehicle can pass and accelerate. If the traffic light sign is red, the vehicle is forced to decelerate and stop there. A vehicle keeping its stride towards a green light will try to stop if the light becomes red before it has passed through the intersection. Therefore a minimum breaking distance $\bar{s}$ is computed by simple kinematic formula [5] as follows.

$$
\bar{s}=v t-\frac{k b}{2} t^{2}=v\left(\frac{v}{k b}\right)-\frac{k b}{2}\left(\frac{v}{k b}\right)^{2}=\frac{v^{2}}{2 k b}
$$

Where $\mathrm{v}$ is the current speed of the vehicle, $\mathrm{t}$ is the time, $\mathrm{kb}$ is the deceleration value. The parameter $\frac{k b}{2} \cdot t^{2}$ represents the maximum safe deceleration i.e., the IDM comfortable braking value $\mathrm{b}$ is scaled by a factor $\mathrm{k}>=1$. If $\bar{s}>\sigma-\mathrm{S}$, the vehicle crosses the intersection at its current speed. Otherwise, it stops by applying a strong adequate deceleration. The second model is Intelligent Driver Model with Lane Changes (IDM-LC) [5]. The model extends the IDM-IM model with the possibility for vehicles to change lane and overtaking behavior. To describe multiple lanes two issues are provided by IDM-LC: first is the separation of traffic flows on different lanes of the same road, second is the overtaking model itself.

In overtaking model MOBIL (Minimizing Overall Braking decelerations Induced by Lane changes) [13] describes the lane changing problem. Lane changes takes place if it satisfies the two conditions i.e., incentive criterion and safety criterion. Incentive criterion means the potential new target lane is more attractive i.e.

$$
a^{l}-a \pm a_{\text {bias }}>p\left[\left(a_{\text {cur }}+a_{\text {new }}\right)-\left(a_{\text {cur }}^{l}+a_{\text {new }}^{l}\right)\right]+a_{t h r}
$$

The model [5] allows a vehicle move to lane 1, when it accelerate, the advantage of the driver who changes its lane $a^{l}-a$, is greater than the disadvantages of the following vehicles in the current $\left(a_{c u r}-a_{c u r}^{l}\right)$ and in the candidate $\left(a_{\text {new }}-a_{\text {new }}^{l}\right)$ lanes. Here $\mathrm{p}$ is a politeness factor whose values are typically less than 1 . To avoid lane skipping in border case an additional lane-changing threshold $a_{t h r}$ has been added. The term $a_{\text {bias }}$ is added to favor movements to one side. On the other hand, safety criterion means the lane change can be performed safely i.e.

$$
a_{\text {new }}^{l}>-a_{\text {safe }}
$$

In safety condition the new back vehicle does not have to brake too hard as a consequence of the lane change. Moreover, the back vehicle's deceleration must be over the safe value ${ }^{a_{\text {safe }}}$.

\section{VEHICULAR MOBILITY MODELS}

Vehicular mobility models are classified in different classes:

\subsection{Synthetic Models}

Synthetic Models [14] wraps all models based on mathematical model. According to Fiore's classification Synthetic models may again be classified in five classes [15]:

\subsubsection{Stochastic Models}

Stochastic model describe mobility descriptions which constrain random movements of nodes on a graph. The movement is random because the vehicles, individually or with group dynamics, follow casual paths over the graph. The vehicles are traveling at randomly chosen speed.

\subsubsection{Traffic Stream Models}

The model considers vehicular mobility as hydrodynamic phenomenon.

\subsubsection{Car-Following Models}

In this model the behavior of each driver is computed on the basis of the vehicle state. The state means its position, speed, and acceleration of the surrounding vehicles.

\subsubsection{Queue Models}

The model deals with vehicles standing in queues and it considers roads as queue buffers.

\subsubsection{Behavioral Models}

In this model each movement of vehicle is determined by behavioral rules. The rules are imposed by social influences, rational decisions or actions following a stimulus-reaction process.

\subsection{Survey-Based Models}

An important source of macroscopic mobility information is survey. In Survey-based Models [15] mobility patterns are extracted from different surveys. These models are used to represent realistic human behavior specifically in urban mesh environments. The inputs of these models are data collected through surveys performed on human activities. Mobility 
simulators implementing survey-based models simulate arrival times (work, lunch time, breaks) and workday times (meeting size, frequency, and duration). The advantage of this model is its ability to represent a particular mobility that would be too complex to model by mathematical equations. Moreover, the main disadvantage of this model is that, the survey data are only able to provide a coarse grain mobility characterization instead of accurate movements.

\subsection{Trace-Based Models}

In Trace-based Models [14] mobility patterns are generated from real mobility traces. Instead of developing complex models and then validating those using mobility traces, a crucial time could be saved by directly extracting generic mobility patterns. These patterns are extracted from movement traces. Mobility traces are gathered through the various measurement campaigns. The main difficult part of this model is to generalize patterns not observed directly by the traces. It is possible to predict mobility patterns not reported in the traces to some extends using complex mathematical equation. Another drawback is the few freely available vehicular traces.

\subsection{Traffic Simulator-Based Models}

In Traffic Simulator-based Models the vehicular mobility traces are extracted from a detailed traffic simulator. The models are made by refining the synthetic models and an intense validation process using real traces or behavior surveys. Parallel Microscopic Simulation of Road Traffic (PARAMICS) [16], Corridor Simulation (CORSIM) [17], Transportation Analysis and Simulation System (TRANSIMS) [18] are able to model urban microscopic traffic monitoring. The disadvantage of this model is the configuration complexity of those traffic simulators as they include a large set of parameters.

\subsection{Urban Vehicular Mobility Models}

In urban vehicular mobility model, street is a crucial factor that forces nodes to confine their movements to well defined paths irrespective of their final destination. Two different urban vehicular mobility models [19] are as follows:

\subsubsection{Stop Sign Model (SSM)}

Stop Sign Model imitates the mobility of vehicles in the presence of stop signs at every intersection. Every vehicle waits for a fixed period of time when it arrives at any intersection before moving to its destination. Every vehicle maintains a specific distance from the vehicle in front of it.

\subsubsection{Traffic Sign Model (TSM)}

In this model vehicle may or may not stop at a traffic light. If a vehicle waits at an intersection, all consecutive vehicles arriving at that intersection wait till it moves. Vehicles queued at any intersection move together after passing the chosen wait time.

\subsection{Street Random Waypoint (STRAW) Mobility} Models

STRAW [20] is a street mobility model that incorporates by simple car following model in real traffic condition. It relies on street plans to build a road map for the specified target region. It also provides at least one lane in each direction on which vehicles can move. It uses a random street placement model to determine the initial positions of vehicles on the field. In street placement model a vehicle places in a lane of a street just before an intersection. If another vehicle is already in that lane, the new vehicle is placed behind the existing one. Two different types of mobility [20] in STRAW are:

\subsubsection{Intrasegment Mobility}

In this mobility model the vehicle changes its speed according to the following rules:

- The vehicle meets an intersection and the next road segment on which it will travel is full. In this case, the vehicle decelerates and stops before the intersection.

- There is a vehicle in front of the current vehicle. In this case, the vehicle decelerates itself to maintain a speed-based following distance between the current vehicle and the vehicle in front of it. The distance formula mentioned in [20]:

$$
S=\alpha+\beta V+\gamma V^{2}
$$

Where, $\mathrm{S}$ is the following distance, $\mathrm{V}$ is the current vehicles speed, $\alpha$ is the vehicle length, $\beta$ is the reaction time, $\gamma$ is the reciprocal of twice the maximum average deceleration of the following vehicle.

- The vehicle meets traffic control mechanism. In this case, the vehicle decelerates before an intersection with a red stoplight or a stop sign; if the stoplight turns green, the vehicle accelerates itself.

- The vehicle turns onto a new road. In this case, the vehicle decelerates before the intersection to make the turn to left or right at a reasonable speed, and then accelerates.

\subsubsection{Intersegment Mobility}

Intersegment Mobility model influences the behavior of vehicles between road segments at intersections. The implementation of vehicular behavior at an intersection is held in two levels. First, mobility model must perform admission control at each intersection. Second, there is a room for the vehicle on the next road segment before it crosses the intersection.

\section{CONCLUSIONS}

VANET is one of the major emerging technologies in wireless communication. A lot of research is being and will be carried out in this field. For measuring the performance of any protocol its simulation is very necessary. But simulation of VANETs 
needs mobility models that reflect the real behavior of vehicular traffic as closely as possible. In this survey different mobility models, their classifications, advantages and disadvantages have described. From the discussion it is clear that currently available mobility models do not reflect all the possible realistic mobility pattern of the vehicles. Therefore, more research work is needed to be done to design a mobility model that captures almost all the possible mobility patterns of the vehicle.

\section{REFERENCES}

[1] J. Haerri, F. Filali and C. Bonnet, "A framewok for mobility models generation and its application to intervehicular networks", in Proc. of 3rd IEEE International Workshop on Mobility Management and Wireless Access, vol.1, June 2005, pp. 42-47.

[2] A.K. Saha and D.B. Johnson, "Modeling Mobility for Vehicular Ad Hoc Networks", in Proc. of the 1st ACM international workshop on Vehicular ad hoc network Rice University, 2004, pp 91-92.

[3] Shrirang Ambaji Kulkarni and G. Raghavendra Rao, "Mobility and Traffic Model Analysis for Vehicular Ad-Hoc Networks", 2010, pp 214-232.

[4] Stefano Fontanelli, "Vehicular Networks: Traffic Simulations and Communication Protocols", University of Pisa, 2008.

[5] M. Fiore, J. Haerri, F. Filali, C. Bonnet, "Vehicular Mobility Simulation for VANETs", in Proc. of the 40th Annual Simulation Symposium, 2007, pp. 301-309.

[6] http://vanet.eurecom.fr.

[7] http://www.ertico.com.

[8] http://www.census.gov/geo/www/tiger.

[9] A. Jardosh, E. Belding-Royer, K. Almeroth, and S. Suri, "Toward realistic mobility models for mobile ad hoc networks", in Proc. of the 9th annual international conference on Mobile computing and networking.2003, pp. 217-229.

[10] http://canu.informatik.uni-stuttgart.de.

[11] I. Seskar, S. Marie, J. Holtzman, J. Wasserman, "Rate of Location Area Updates in Cellular Systems", in Proc. of VTC, vol.2, 1992, pp. $694-697$.

[12] M. Trieber, A. Hennecke, D. Helbing, "Congested traffic states in empirical observations and microscopic simulations", Physics Review E, vol. 62, 2000, pp. 1805-1824.

[13] http://vwi.tudresden.de/treiber/MicroApplet/MOBIL.html

[14] Jobin Mathew, "Mobility Model for Vehicular Ad Hoc Networks: a survey and taxonomy", Department of computer Science, COCHIN University. June 2010.

[15] J. Haerri, F. Filali and C. Bonnet, "Mobility Models for Vehicular Ad Hoc Networks: A Survey and Taxonomy", IEEE Communications Surveys \& Tutorials, vol. 11, issue 4, 2009, pp 19-41.

[16] http://www.Paramics-online.com.
[17] http://www.mctrans.ce.ufl.edu/featured/TSIS/Version5/ corsim.htm.

[18] http://transims.tsasa.lanl.gov.

[19] Atulya Mahajan, Niranjan Potnis, Kartik Gopalan and An-I A. Wang, "Urban Mobility Models for VANETs", Computer Science, Florida State University. 2006, pp. 1-8.

[20] David R. Choffnes, Fabian E. Bustamante "STRAW An Integrated Mobility and Traffic Model for VANETs", Department of Computer Science, Northwest University, 2005. 